\title{
Hausdorff dimension for horseshoes
}

\author{
HEATHER MCCLUSKEY AND ANTHONY MANNING \\ Mathematics Institute, University of Warwick, Coventry CV4 7AL, England
}

(Received 20 October 1982)

Abstract. We shall measure how thick a basic set of a $C^{1}$ axiom $A$ diffeomorphism of a surface is by the Hausdorff dimension of its intersection with an unstable manifold. This depends continuously on the diffeomorphism. Generically a $C^{2}$ diffeomorphism has attractors whose Hausdorff dimension is not approximated by the dimension of its ergodic measures.

\section{Introduction}

The Smale horseshoe [16] is an invariant set $\Lambda$ (a 'basic set') of a certain axiom $A$ diffeomorphism of a surface. It behaves like a saddle in that many orbits approach for a while and then leave a neighbourhood of $\Lambda$. Bowen and Ruelle [2], [5], showed that, for $C^{2}$ diffeomorphisms $f$ satsifying axiom $A$, the set $W^{s}(\Lambda)$ of points that do approach $\Lambda$ has Lebesgue measure zero. However, [3] exhibits a $C^{1}$ diffeomorphism where $W^{s}(\Lambda)$ has positive Lebesgue measure. Here we consider a more delicate way to describe how substantial the basic set $\Lambda$ is. The unstable manifold $W^{u}(x)$ of a point $x$ of $\Lambda$ meets it in a Cantor set. We calculate the Hausdorff dimension of this set and show that it is independent of $x$ and varies continuously with the $C^{1}$ diffeomorphism $f$. We also show that, generically, a $C^{2}$ diffeomorphism has attractors whose Hausdorf dimension is not approximated by the dimension of its ergodic measures! Finally we consider the bifurcation from Anosov to DA and show that the Hausdorff dimension of the attractor is continuous across the bifurcation.

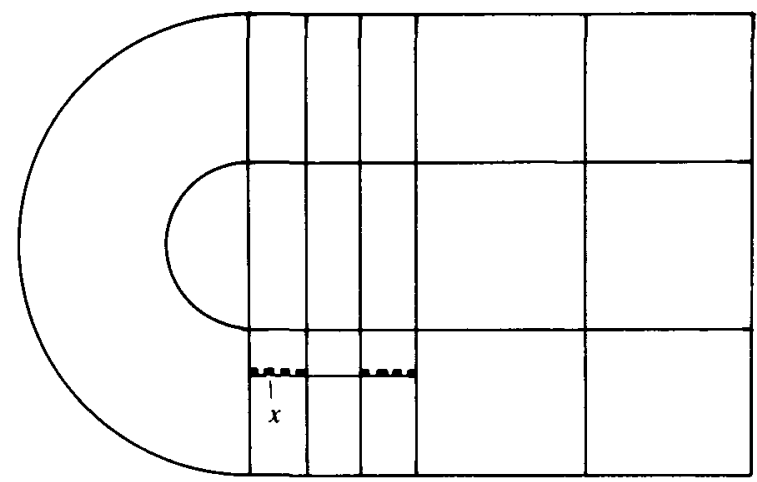

FIGURE 1

1. Hausdorff dimension in an unstable manifold

Let $\boldsymbol{M}=\boldsymbol{M}^{2}$ be a compact surface without boundary and let $f \in \operatorname{Diff}^{1}(\boldsymbol{M})$ satisfy axiom $A$ and the No Cycle Property (which are sufficient conditions for $\Omega$-stability 
[17]). Let $\Lambda$ be one of the basic sets for $f$ and let $\Lambda(g)$ be the corresponding basic set for $g$ in some $C^{1}$ neighbourhood of $f$. We denote by $W_{\mathrm{loc}}^{u}(x)$ and $W_{\mathrm{loc}}^{u}(x, g)$ the local unstable manifolds of a point $x$ under the diffeomorphisms $f$ and $g$.

Consider the set

$$
W_{\text {loc }}^{u}(x, g) \cap \Lambda(g) .
$$

We may regard the Hausdorff dimension of this set as a measure of the 'fatness' of the basic set $\Lambda(\mathrm{g})$ in the unstable direction and hence of its influence on the nearby dynamics. Hausdorff dimension is defined for a metric space $X$ as follows, see [10, chapter 7]. For a non-negative real number $p$ the Hausdorff $p$-measure of $X$ is

$$
m_{p}(X)=\sup _{\varepsilon>0} \inf \sum_{i=1}^{\infty}\left[\operatorname{diam}\left(A_{i}\right)\right]^{p}
$$

where the infimum is taken over all covers of $X$ by a countable collection of subsets $A_{1}, A_{2}, A_{3}, \ldots$, each of diameter less than $\varepsilon$. Then the Hausdorff dimension $\operatorname{HD}(X)$ is defined by

$$
\operatorname{HD}(X)=\sup \left\{p ; m_{p}(X)=\infty\right\}=\inf \left\{p ; m_{p}(X)=0\right\} .
$$

We shall also need the pressure of a continuous function [14], [18]. Let $C(\Lambda, \mathbb{R})$ denote the continuous real-valued functions on the basic set $\Lambda$ of $f$ with the sup norm. Then pressure $P: C(\Lambda, \mathbb{R}) \rightarrow \mathbb{R}$ is defined by

$$
P(\psi)=\limsup _{n \rightarrow \infty} n^{-1} \log \sup _{E} \sum_{x \in E} \exp \left(\sum_{i=0}^{n-1} \psi\left(f^{i} x\right)\right),
$$

where the supremum is taken over $(n, \delta)$-separated sets $E$ and $\delta$ is an expansive constant for $f \mid \Lambda$. The variational principle [18] gives an alternative description of pressure as

$$
P(\psi)=\sup _{\mu}\left\{h_{\mu}(f)+\int \psi d \mu\right\}
$$

where the supremum is taken over $f$-invariant Borel probability measures $\mu$. From this it follows easily, or see [18], that

$$
|P(\phi)-P(\psi)| \leq\|\phi-\psi\|,
$$

$P$ is convex and, for a strictly negative function $\psi$, the map

$$
t \mapsto P(t \psi)
$$

is strictly decreasing.

Now we can state our first theorem.

THEOREM 1. Let $\Lambda$ be a basic set for a $C^{1}$ axiom A diffeomorphism $f: M^{2} \rightarrow M^{2}$ with $(1,1)$ splitting

Define $\phi: W^{u}(\Lambda) \rightarrow \mathbb{R}$ by

$$
T_{\Lambda} M=E^{s} \oplus E^{u} .
$$

$$
\phi(x)=-\log \left\|D f_{x} \mid E_{x}^{u}\right\| .
$$

Then the Hausdorff dimension of $W^{u}(x) \cap \Lambda$ is given by the unique $\delta$ for which

$$
P_{f \mid \Lambda}(\delta \phi)=0 \text {. }
$$


$\delta$ is independent of $x \in \Lambda$, and $0 \leq \delta \leq 1$. Moreover, $\delta$ depends continously on $f$ in the $C^{1}$ topology on diffeomorphisms.

Proof. For purposes of calculating Hausdorff dimension it does not matter which Riemannian metric we choose on $M$ since any two are Lipschitz equivalent so let us choose a metric 'adapted' to $f,[12]$. Now $\phi$ is a negative function and so $t \mapsto P(t \phi)$ is strictly monotonically decreasing, [18].

$$
P(0)=h(f \mid \Lambda) \geq 0
$$

and, for $C^{2}$ diffeomorphisms $f$, we have $P(\phi) \leq 0$ by [2]. Hence there is a unique value $\delta$ of $t$ for which $P(t \phi)=0$ and, at least for $f$ of class $C^{2}$, it satisfies $0 \leq \delta \leq 1$.

Given any $t>\delta$ choose $\varepsilon>0$ such that

$$
P(t(\phi+\varepsilon))<0 \text {. }
$$

Choose a Markov partition $\mathcal{U}$ for $f \mid \Lambda$ so fine that $\phi$ varies by at most $\varepsilon$ on the intersection of $W^{u}(\Lambda)$ with the region of $M$ enclosed by each rectangle. We also need each rectangle to be enclosed by four intervals of stable and unstable manifold and we do not allow rectangles to consist of finitely or infinitely many pieces between each other. (This 'connected' or 'convex' property can be achieved for our two-dimensional case by modifying the construction given in [ $2, \mathrm{p} 81]$ to divide $T_{i}$ into up to nine parts for each neighbour $T_{k}$ as in figure $2(a)$ instead of four as in figure 2(b). Alternatively see [15].)
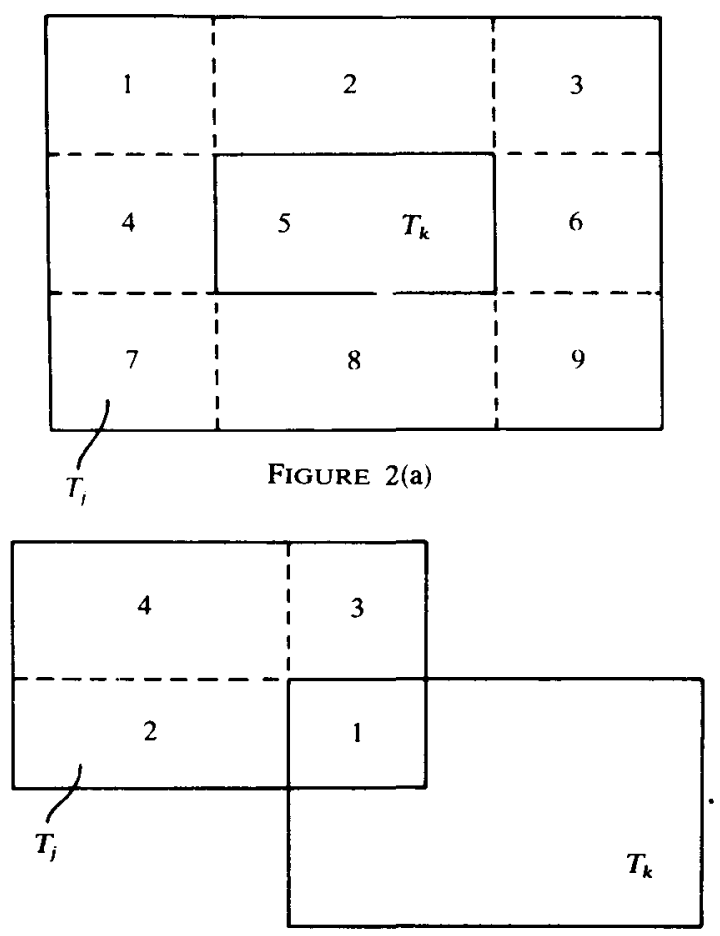

FIGURE 2(b) 
Consider a compact set

$$
W=W_{\text {loc }}^{u}(x) \cap \Lambda \text {. }
$$

We shall show that the Hausdorff $t$-measure of $W$ is zero. Since each unstable manifold meets $\Lambda$ in a countable union of such pieces it cannot then have dimension more than $\delta$.

The compact set $W$ is in a $C^{1}$ submanifold of dimension one and the metric on that as a Riemannian manifold in its own right is Lipschitz equivalent to the restriction to $W$ of the metric on $M$. Thus the Hausdorff dimension of $W$ will be the same for the two metrics and we may choose to work with the former.

Choose some large $n$ and consider the cover $u_{n}$ of $W$ by $n$-cylinders. These are intervals $I$ so that, for each $i$ with $0 \leq i \leq n$, all $y$ in $I$ have $f^{i} y$ in the same rectangle. By the Mean Value Theorem there is some particular $y \in I$ for which

$$
\operatorname{diam} I=\operatorname{diam}\left(f^{n} I\right) \cdot\left\|D f_{y}^{n} \mid E^{u}\right\|^{-1}=\operatorname{diam}\left(f^{n} I\right) \cdot \exp \sum_{i=0}^{n-1} \phi\left(f^{i} y\right) .
$$

This point $y$ could be wandering so choose some $z \in I \cap \Lambda$. Then

$$
\exp \sum_{i=0}^{n-1} \phi\left(f^{i} y\right) \leq \exp \sum_{i=0}^{n-1}\left(\phi\left(f^{i} z\right)+\varepsilon\right) .
$$

Now $f^{n} I$ stretches across some rectangle so

$$
\operatorname{diam} I \leq m \exp \sum_{i=0}^{n-1}\left(\phi\left(f^{i} z\right)+\varepsilon\right)
$$

where the constant $m$ is the maximum width of any rectangle.

Let $E$ be the set of these points $z$ chosen one from each interval $I$ in the cover $U_{n}$ of $W$ by $n$-cylinders. The set $E$ is arranged in some order along $W$ and we write

$$
E=E_{1} \cup E_{2}
$$

where $E_{1}$ and $E_{2}$ are the odd and even numbered points in this order in $E$. Now $E_{1}$ and $E_{2}$ are both $(n+1, \delta)$-separated sets where $\delta$ is the minimum distance apart of any two disjoint rectangles. This uses the 'convexity' property of the rectangles. For the cover $\mathcal{U}_{n}$ of $W$ we find

$$
\begin{aligned}
\sum_{I \in \mathcal{Q}_{n}}(\operatorname{diam} I)^{t} & \leq m^{t} \sum_{z \in E} \exp \sum_{i=0}^{n-1} t\left(\phi\left(f^{i} z\right)+\varepsilon\right) \\
& \leq 2 m^{t} \max _{i=1,2} \sum_{z \in E_{i}} \exp \sum_{i=0}^{n-1} t\left(\phi\left(f^{i} z\right)+\varepsilon\right) \\
& \leq 2 m^{t} \sup _{E^{\prime}} \sum_{x \in E^{\prime}} \exp \sum_{i=0}^{n-1} t\left(\phi\left(f^{i} x\right)+\varepsilon\right) \\
& \leq 2 m^{t} c \sup _{E^{\prime}} \sum_{x \in E^{\prime}} \exp \sum_{i=0}^{n} t\left(\phi\left(f^{i} x\right)+\varepsilon\right),
\end{aligned}
$$

where $c$ is a constant involving the norm of $\phi$ and the supremum is taken over $(n+1, \delta)$-separated sets $E^{\prime}$. Now this expression has the negative growth rate $P(t(\phi+\varepsilon))$ as $n$ increases so the Hausdorff $t$-measure of $W$ is zero as claimed and the dimension of $W$ is at most $\delta$. 
On the other hand consider $t=\delta . P(\delta \phi)=0$ and $\delta \phi$ has an ergodic equilibrium state $\mu$, say, (which need not be unique when $f$ is $C^{1}$ ). Thus

$$
h_{\mu}+\delta \mu(\phi)=0 \text {. }
$$

By [11] the subset of $W$ consisting of points that are future generic for $\mu$ has Hausdorff dimension $h_{\mu} / \chi_{\mu}$ where $\chi_{\mu}$ is the positive Lyapunov exponent for $\mu$. But $\chi_{\mu}$ is just $-\mu(\phi)$ so this subset of $W$ has Hausdorff dimension

$$
-h_{\mu} / \mu(\phi)=\delta
$$

and the dimension of $W$ is precisely $\delta$. This proof is different from [4, lemma 10] because our $\phi$ need not have any Gibbs state.

It remains to prove that $\delta$ depends continuously on $f$ in the $C^{1}$ topology. That will then imply that $\delta \leq 1$ for $f C^{1}$ since this holds for $C^{2}$ diffeomorphisms and they are dense in the $C^{1}$ topology. For any $g$ close enough to $f$ in the $C^{1}$ topology there is a homeomorphism $h: \Lambda \rightarrow \Lambda(g)$ satisfying $h f=g h$. Now $h$ only moves points a small distance and so $D g_{h x}$ is near $D f_{h x}$, since $g$ is $C^{1}$ near $f$, and this is near $D f_{x}$, since $f$ is $C^{1}$. To compare $E^{u}(g)$ on $\Lambda(g)$ with $E^{u}$ on $\Lambda$ consider a family of cones in $T_{\Lambda} M$ containing $E^{u}$, invariant by $D f$. Extend this to a neighbourhood of $\Lambda$ still invariant by $D f$. This family is invariant by $D g$ and so contains $E^{u}(g)$. Hence the map

$$
\phi_{g} h: \Lambda \rightarrow \mathbb{R} \quad x \mapsto-\log \left\|D g_{h x} \mid E^{u}(g)\right\|
$$

is close to $\phi: \Lambda \rightarrow \mathbb{R}$. The conjugacy $h$ makes $P_{g}\left(t \phi_{g}\right)$ vanish for the same value of $t$ as $P_{f}\left(t\left(\phi_{g} h\right)\right)$ and this is near the value $\delta$ that makes $P(t \phi)$ vanish since $P: C(\Lambda, \mathbb{R}) \rightarrow \mathbb{R}$ is continuous. Thus the Hausdorff dimension of $W^{u}(h x, g) \cap \Lambda(g)$ is near that of $W^{u}(x, f) \cap \Lambda$ for $g C^{1}$ near $f$.

From now on let $\delta$ and $\phi$ be denoted by $\delta_{u}$ and $\phi^{u}$. In [3] Bowen constructed a $C^{1}$ diffeomorphism with a horseshoe of positive measure. This horseshoe is a product of sets of positive Lebesgue measure in a stable and an unstable manifold and so has $\delta_{u}=1$. We now show how unusual this is.

Corollary 1. For a $C^{1}$ open dense subset of the $C^{1}$ axiom $A$ no-cycle diffeomorphisms of a surface each basic set that is not an attractor has $\delta_{u}<1$.

Proof. The property holds for a $C^{2}$ diffeomorphism $f$ by [2, theorem 4.11$]$ which says that

$$
P\left(\phi^{u}\right)<0
$$

if the basic set is not an attractor. By the continuity property in theorem 1 we still have $\delta_{u}<1$ for a $C^{1}$ neighbourhood of $f$. The union of these neighbourhoods gives the required $C^{1}$ open dense set.

COROllary 2. In the situation of theorem 1 the Hausdorff dimension of $W^{s}(x) \cap \Lambda$ is the unique number $\delta_{s}, 0 \leq \delta_{s} \leq 1$, for which

$$
P\left(\delta_{s} \phi^{s}\right)=0
$$

where $\phi^{s}: \Lambda \rightarrow \mathbb{R}$ is the negative function given by

$$
\phi^{s}(y)=\log \left\|D f \mid E_{y}^{s}\right\| .
$$

Proof. Apply theorem 1 to $f^{-1}$. 
COROllary 3. The Hausdorff dimension $\delta_{u}$ in theorem 1 satisfies

$$
-h_{\text {top }} / m\left(\phi^{u}\right) \leq \delta_{u} \leq h_{\text {top }} /\left(h_{\text {top }}-P\left(\phi^{u}\right)\right),
$$

where $h_{\text {top }}$ is the topological entropy of $f \mid \Lambda$ and $m\left(\phi^{u}\right)$ is the integral of $\phi^{u}$ with respect to the measure of maximal entropy.

Proof. The variational inequality for pressure gives

$$
\begin{aligned}
0 & =P\left(\delta_{u} \phi^{u}\right)=\sup _{\nu}\left(h_{\nu}+\delta_{u} \nu\left(\phi^{u}\right)\right) \\
& \geq h_{m}+\delta_{u} m\left(\phi^{u}\right)=h_{\mathrm{top}}+\delta_{u} m\left(\phi^{u}\right)
\end{aligned}
$$

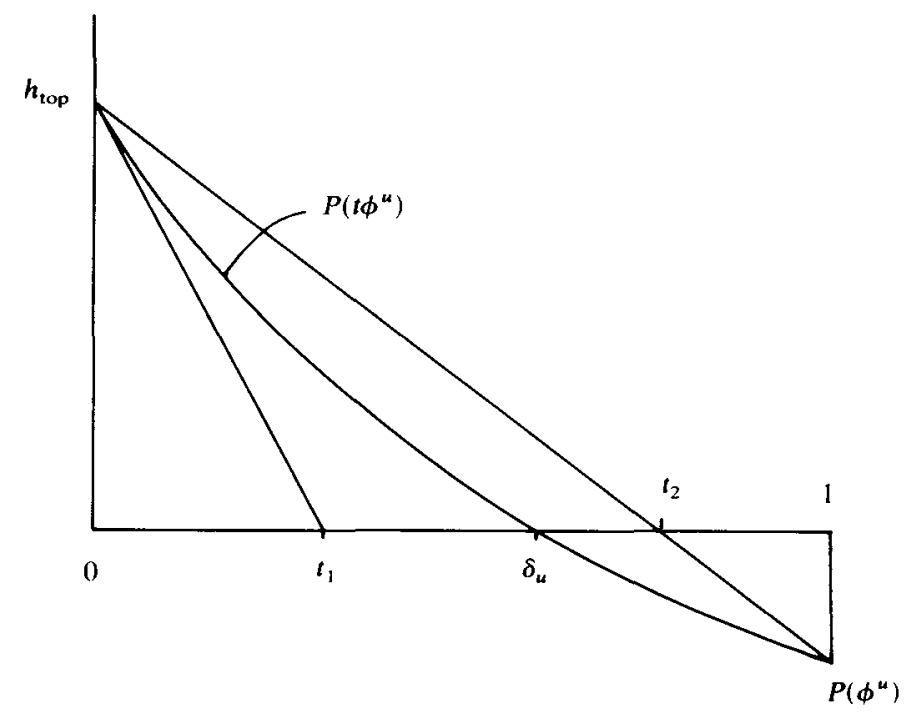

Figure 3

from which we have our first inequality. The second comes from the convexity, whereby

$$
t_{2}=h_{\text {top }} /\left(h_{\text {top }}-P\left(\phi^{u}\right)\right) \geq \delta_{u} .
$$

Remark. Notice that $m\left(\phi^{u}\right)<0$, that $m$ is the unique equilibrium state of the zero function, and that $P\left(\phi^{u}\right) \leq 0$. Indeed, Bowen and Ruelle showed that $P\left(\phi^{u}\right)$ has the following significance for $C^{2}$ diffeomorphisms [2]. The Lebesgue measure of the set of those points whose orbits remain within $\varepsilon$ of $\Lambda$ from time 0 to $n$ decays like $\exp n P\left(\phi^{u}\right)$. Since the pressure curve

$$
t \mapsto P\left(t \phi^{u}\right)
$$

is convex there is no precise relation between $\delta_{u}$ and $P\left(\phi^{\prime \prime}\right)$ although they both indicate the thickness of $\Lambda$. In fact the variational inequality shows that the straight lines

$$
t \mapsto h_{\text {top }}+t m\left(\phi^{u}\right) \quad \text { and } \quad t \mapsto h_{\mu}+t \mu\left(\phi^{u}\right),
$$

where $\mu$ is an equilibrium state for $\phi$, lie below the curve $t \mapsto P(t \phi)$. They are actually tangents to the pressure curve at $t=0$ and 1 provided the curve is differentiable there. The upper and lower bounds for $\delta_{u}$ in corollary 3 are now equal precisely 
when

$$
m\left(\phi^{u}\right)=\mu\left(\phi^{u}\right)
$$

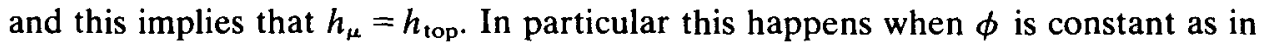
the case of an algebraic Anosov diffeomorphism of $T^{2}$.

\section{Hausdorff dimension of invariant measures}

Recently, Young [20] has proposed to define the Hausdorff dimension $\operatorname{HD}(\mu)$ of a probability measure $\mu$ as

$$
\inf \{\mathrm{HD}(Y) ; \mu(Y)=1\}
$$

She proves:

THEOREM (Young). Let $f: M^{2} \rightarrow M^{2}$ be a $C^{2}$ diffeomorphism or a $C^{1}$ axiom $A$ diffeomorphism. Let $\mu$ be an f-invariant ergodic Borel probability measure with Lyapunov exponents $\lambda_{1}>0>\lambda_{2}$. Then

$$
\mathrm{HD}(\mu)=h_{\mu}\left(1 / \lambda_{1}-1 / \lambda_{2}\right) \text {. }
$$

We shall investigate the relationship of this to the Hausdorff dimension of the basic set on which $\mu$ is concentrated. Let $\Lambda$ be a basic set of a $C^{2}$ axiom $A$ diffeomorphism of a surface. Assume that $\Lambda$ is not just a periodic source or sink in which case it has $E^{s}$ and $E^{u}$ one-dimensional. Young's formula gives

$$
\operatorname{HD}(\mu)=-h_{\mu}\left(1 / \mu\left(\phi^{u}\right)+1 / \mu\left(\phi^{s}\right)\right)
$$

for an ergodic measure $\mu$ on $\Lambda$. Now the canonical coordinates are Lipschitz [9] and $\Lambda$ is locally Lipschit $z$ equivalent to a product of metric spaces

$$
\left(W_{\text {loc }}^{u}(x) \cap \Lambda\right) \times\left(W_{\text {loc }}^{s}(x) \cap \Lambda\right) .
$$

By [1, theorem 2] and [4, lemma 10], the Hausdorff dimension of this product is

$$
\delta_{u}+\delta_{s}
$$

Pesin has alşo considered this question in [13].

Let $\mu_{u}, \mu_{s}$ denote respectively the unique equilibrium states of $\delta_{u} \phi^{u}$ and $\delta_{s} \phi^{s}$. Since

$$
P\left(\delta_{u} \phi^{u}\right)=0
$$

we have

$$
h_{\mu}+\delta_{u} \mu\left(\phi^{u}\right) \leq 0 \text { or }-h_{\mu} / \mu\left(\phi^{u}\right) \leq \delta_{u}
$$

with equality only for $\mu=\mu_{u}$. Similarly

$$
-h_{\mu} / \mu\left(\phi^{s}\right) \leq \delta_{s}
$$

with equality only for $\mu=\mu_{s}$. Adding the inequalities we obtain

$$
\operatorname{HD}(\mu) \leq \delta_{u}+\delta_{s}=\operatorname{HD}(\Lambda)
$$

with equality only if $\mu=\mu_{u}=\mu_{s}$. As in [2, corollary 4.15], $\mu_{u}=\mu_{s}$ fails for $f$ in a $C^{2}$ open dense set. Now

$$
-\left(1 / \mu\left(\phi^{u}\right)+1 / \mu\left(\phi^{s}\right)\right)
$$

is a positive continuous function of $\mu$ and $h_{\mu}$ is an upper semicontinuous function of $\mu$ (since $f \mid \Lambda$ is expansive [6]) so $\operatorname{HD}(\mu)$ depends upper semi-continuously on 
$\mu$ and attains its supremum. Thus, if $\mu_{u} \neq \mu_{s}$,

$$
\sup _{\mu} \operatorname{HD}(\mu)<\operatorname{HD}(\Lambda) \text {. }
$$

As in the proof of theorem 1, for $g$ in some prescribed neighbourhood of $f$ we have

$$
\left\|\phi_{g}^{u} \circ h-\phi^{u}\right\|<\varepsilon \text { and }\left\|\phi_{g}^{s} \circ h-\phi^{s}\right\|<\varepsilon
$$

where $h: \Lambda \rightarrow \Lambda_{g}$ is the conjugacy. Then

$$
\left|\frac{1}{\left(h_{*} \mu\right)\left(\phi_{g}^{u}\right)}-\frac{1}{\mu\left(\phi^{u}\right)}\right| \leq \frac{\varepsilon}{\mu\left(\phi_{g}^{u} h\right) \mu\left(\phi^{u}\right)} \leq \frac{\varepsilon}{\inf \left|\phi^{u}\right| \cdot\left(\inf \left|\phi^{u}\right|-\varepsilon\right)} .
$$

Hence $\sup _{\mu} \operatorname{HD}(\mu)$ depends continuously on the $C^{2}$ diffeomorphism $f$. We can summarise this as:

THEOREM 2. Let $f: \Lambda \rightarrow \Lambda$ be a basic set of a $C^{2}$ axiom $A$ diffeomorphism of a surface. Then

$$
\mathrm{HD}(\Lambda)=\delta_{u}+\delta_{s}
$$

varies continuously with $f$. So does $\sup _{\mu} \operatorname{HD}(\mu)$ taken over ergodic measures $\mu$ on $\Lambda$ and the former is strictly greater than the latter except on the nowhere dense set of those diffeomorphisms for which $\mu_{s}=\mu_{u}$.

Remark. Yorke's suggested formula [7] for the dimension or capacity of an attractor with exponents $\lambda_{1}>0>\lambda_{2}$ (with $\lambda_{1}+\lambda_{2}<0$ ) was $1-\left(\lambda_{1} / \lambda_{2}\right)$ and when $\lambda_{1}, \lambda_{2}$ are calculated for the Bowen-Ruelle measure $\nu$ this agrees with Young's formula for

$$
\operatorname{HD}(\nu)=h_{\nu}\left(1 / \lambda_{1}-1 / \lambda_{2}\right)
$$

since here $\lambda_{1}=h_{\nu}$ by $P\left(\phi^{u}\right)=0$. (See [20] for discussion of the relationship between capacity and Hausdorff dimension.) Thus our theorem 2 says that for an open dense set of $C^{2}$ axiom $A$ diffeomorphisms of surfaces Yorke's formula gives too low an answer. One would expect to observe this, for example, for an Anosov diffeomorphism of the torus which is a perturbation of a linear one and has $\phi^{s}$ constant but $\phi^{\prime \prime}$ not. An invariant subset of an attractor that does have full dimension is

$$
\left\{x \in \Lambda ; \frac{1}{n} \sum_{i=0}^{n-1} \delta_{f^{i} x} \rightarrow \nu \text { and } \frac{1}{n} \sum_{i=-n+1}^{0} \delta_{f^{i} x} \rightarrow \nu^{-} \quad \text { as } n \rightarrow \infty\right\}
$$

where $\nu^{-}$is the equilibrium state for $\delta_{s} \phi^{s}$. Gurevich and Oseledec put a dissipative measure here in $[\mathbf{8}]$.

\section{Continuity across a bifurcation}

By theorem 2 an attractor of a $C^{2}$ Axiom $A$ diffeomorphism of a surface such as a DA attractor [19] has Hausdorff dimension $1+\delta_{s}$ and by theorem 1 this varies continuously with $f$. So we can ask whether the dimension of the attractor is continuous across the bifurcation from Anosov to DA.

Let $f_{a}, 0 \leq q \leq 1$ be a path through $C^{2}$ diffeomorphisms of the torus $T^{2}$ starting at a linear Anosov diffeomorphism $f_{0}$ and ending at a DA example $f_{1}$ with every $f_{q}$ agreeing with $f_{0}$ outside a fixed neighbourhood $V$ of the origin. Assume that each $f_{q}$ preserves the stable manifolds of $f_{0}$ which are then its own stable manifolds (except for splitting the line though the origin in two). 

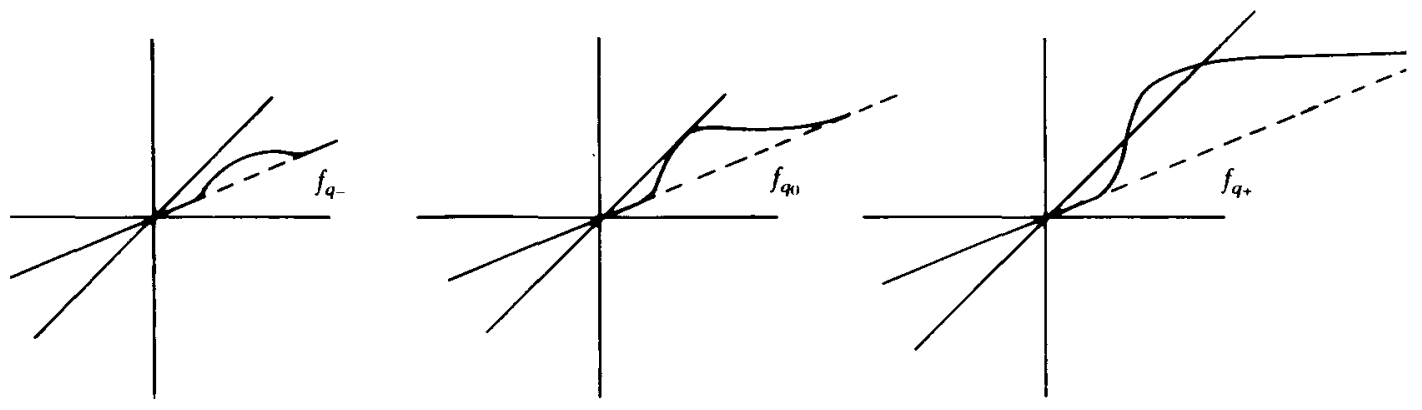

$f_{4} \mid L$

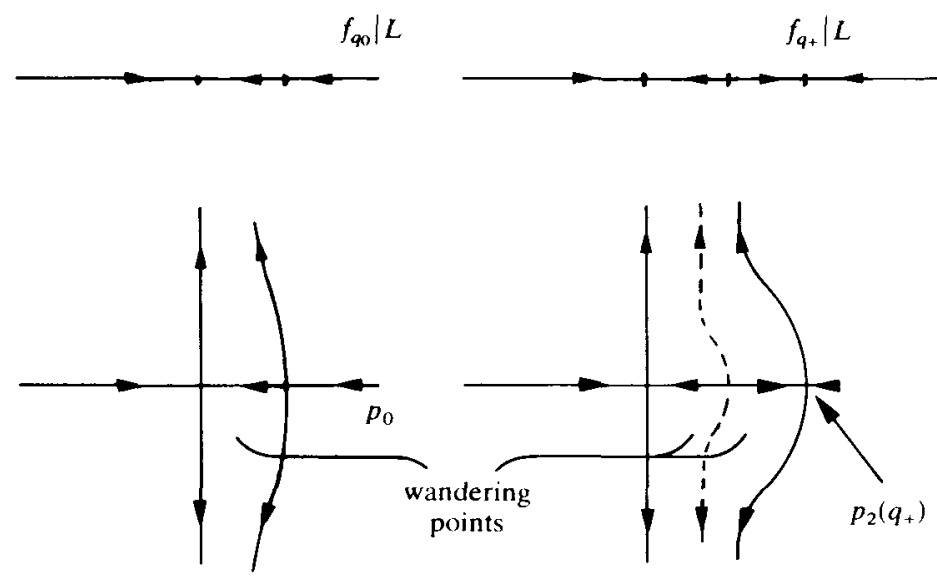

Figure 4

Let $L$ denote $W_{\varepsilon}^{s}\left(0, f_{0}\right)$ and consider $f_{q} \mid L$. Figure 4 shows the graph of $f_{q} \mid L$ for three values $q_{-}<q_{0}<q_{+}$of $q$ where $q_{0}$ is the bifurcation value. It also shows below these graphs the behaviour of $f_{q}$ on $L$ and on a neighbourhood of the origin. The saddle-node fixed point $p$ of $f_{q_{0}}$ is fairly near 0 in $L$.

THEOREM 3. Under the above hypotheses on $f_{q}, 0 \leq q \leq 1$, the Hausdorff dimension of $\Omega\left(f_{q}\right)$ is a continuous function of $q$.

Proof. We have already proved continuity for $q<q_{0}$ and for $q>q_{0}$. So it remains to prove that the dimension tends to 2 as $q \rightarrow q_{0}+$ and that $\Omega\left(f_{q_{0}}\right)$ has dimension 2. For the first of these note that the new fixed point $p_{2}\left(q_{+}\right)$has its contracting eigenvalue near 1 . Specifically,

$$
\left(f_{q} \mid L\right)^{\prime}\left(p_{2}(q)\right) \rightarrow 1
$$

from below as $q \rightarrow q_{0}+$. The delta measure at this fixed point is invariant, has zero entropy and integrates $\phi_{q}^{s}$ to give $\phi_{q}^{s}\left(p_{2}(q)\right)$ which tends to 0 as $q \rightarrow q_{0}+$. Now

$$
P_{f_{q}}\left(\phi_{q}^{s}\right)=\sup _{\mu}\left(h_{\mu}\left(f_{q}\right)+\mu\left(\phi_{q}^{s}\right)\right) \geq \phi_{q}^{s}\left(p_{2}(q)\right) \rightarrow 0 \quad \text { as } q \rightarrow q_{0}+.
$$

Hence $\delta_{s} \rightarrow 1$ as $q \rightarrow q_{0}+$ and so the dimension of $\Omega\left(f_{q}\right)$, which by theorem 2 is $1+\delta_{s}$, tends to 2 as $q \rightarrow q_{0}+$ as required. 
For the second statement notice that $\Omega\left(f_{q_{0}}\right)$ is the complement of the open half-space to the left of $p_{0}$, which consists of points that wander away from $p_{0}$. The dimension of $\Omega\left(f_{q_{0}}\right)$ in a stable manifold is at least the $\delta_{s}$ associated with a hyperbolic invariant subset $\Lambda$ of $\Omega$ obtained by deleting the orbit of a very small open rectangle containing $p_{0}$. This $\delta_{s}$ is arbitrarily close to 1 because the pressure of $\phi_{q_{0}}^{s}$ on $\Lambda$ can be seen to be arbitrarily near 0 by considering the invariant atomic measure on a periodic orbit in $\Lambda$ that spends most of its orbit near the fixed point $p_{0}$ at which $\phi_{q_{0}}^{s}$ is 0 . Thus $\Omega\left(f_{q}\right)$ has Hausdorff dimension 2 at $q=q_{0}$ and the dimension is a continuous function of $q$.

\section{REFERENCES}

[1] A. S. Besicovitch \& P. A. P. Moran. The measure of product and cylinder sets. J. Lond. Math. Soc. 20 (1945), 110-120.

[2] R. Bowen. Equilibrium states and the ergodic theory of Anosov diffeomorphisms. Lecture Notes in Mathematics No. 470. Springer Verlag: New York, 1975.

[3] R. Bowen. A horseshoe with positive measure. Inv. Math. 29 (1975), 203-204.

[4] R. Bowen. Hausdorff dimension of quasi-circles. Publ. Math. IHES. 50 (1979), 11-25.

[5] R. Bowen \& D. Ruelle. The ergodic theory of axiom $A$ flows. Inv. Math. 29 (1975), 181-202.

[6] M. Denker, C. Grillenberger \& K. Sigmund. Ergodic theory on compact spaces. Lecture Notes in Mathematics No. 527. Springer Verlag: Berlin, 1976.

[7] P. Frederickson, J. Kaplan \& J. Yorke. The dimension of the strange attractor for a class of difference systems. Preprint (1980).

[8] B. M. Gurevič \& V. I. Oseledeč. Gibbs distributions and dissipativeness of $U$-diffeomorphisms. Sov. Math. Dokl. 14 (1973), 570-573.

[9] M. Hirsch \& C. Pugh. Stable manifolds and hyperbolic sets. In Global Analysis. Proc. Symp. Pure Math. 14 (1970), 133-163.

[10] W. Hurewicz \& H. Wallman. Dimension Theory. Princeton University Press: Princeton, 1941.

[11] A. Manning. A relation between Lyapunov exponents, Hausdorff dimension and entropy. Ergod. Th. \& Dynam. Sys. 1 (1981), 451-459.

[12] Z. Nitecki. Differentiable Dynamics. MIT Press: 1971.

[13] Ya. B. Pesin. A formula for the Hausdorff dimension of a two-dimensional hyperbolic attractor. Funk. Anal. i ego Prilozh. 16 (1982), no. 4, 82-83.

[14] D. Ruelle. Thermodynamic formalism. Addison-Wesley: Reading, 1978.

[15] Ya. G. Sinai. The construction of Markov partitions. Func. Anal. Appl. 2 (1968), 70-80.

[16] S. Smale. Differentiable dynamical systems. Bull. Amer. Math. Soc. 73 (1967), 747-817.

[17] S. Smale. The $\Omega$-stability theorem. In Global Analysis, Proc. Symp. Pure Math. 14 (1970), 289-297.

[18] P. Walters. A variational principle for the pressure of continuous transformations. Amer. J. Math. 97 (1976), 937-971.

[19] R. F. Williams. The DA maps of Smale and structural stability. In Global Analysis, Proc. Symp. Pure Math. 14 (1970), 329-334.

[20] L. S. Young. Dimension, entropy and Lyapunov exponents. Ergod. Th. \& Dynam. Syst. 2 (1982), 109-124. 